\title{
Aproximación al perfil no profesional del micro instagramer de moda en España
}

\section{Exploration of the nonprofessional profile of the fashion micro instagramer in Spain}

\author{
Ángela Agulleiro-Prats; Susana Miquel-Segarra; Irene García-Medina; Zahaira- \\ Fabiola González-Romo
}

Cómo citar este artículo:

Agulleiro-Prats, Ángela; Miquel-Segarra, Susana; García-Medina, Irene; González-Romo, Zahaira-Fabiola (2020). "Aproximación al perfil no profesional del micro instagramer de moda en España". Profesional de la información, v. 29, n. 4, e290439.

https://doi.org/10.3145/epi.2020.jul.39

Artículo recibido el 19-12-2019

Aceptación definitiva: 01-04-2020

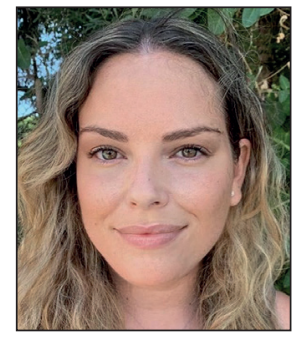

Ángela Agulleiro-Prats

https://orcid.org/0000-0002-5223-0380

Universitat Jaume I

Avinguda de Vicent Sos Baynat, s/n

12071 Castelló de la Plana, España

angela.agulleiro@alumail.uji.es

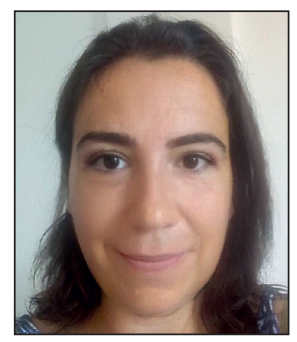

Irene García-Medina

https://orcid.org/0000-0003-4424-3357

Glasgow Caledonian University

Cowcaddens Rd,

Glasgow G4 OBA, Reino Unido

irene.garcia2@gcu.ac.uk

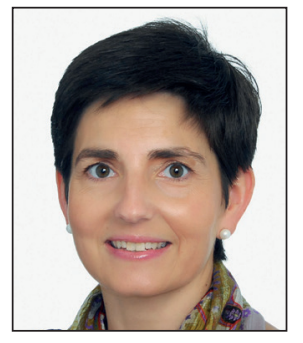

Susana Miquel-Segarra https://orcid.org/0000-0002-0337-7503

Universitat Jaume I

Avinguda de Vicent Sos Baynat, s/n 12071 Castelló de la Plana, España smiquel@uji.es

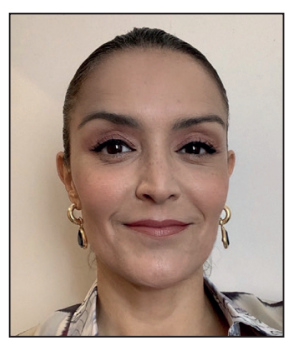

Zahaira-Fabiola González-Romo https://orcid.org/0000-0003-2034-9704

Universitat Internacional de Catalunya Immaculada, 22. 08017 Barcelona, España zfgonzalez@uic.es $\bowtie$

\section{Resumen}

La evolución de las redes sociales en general y de Instagram en particular, ha propiciado el nacimiento y la proliferación de los influenciadores digitales, una figura que toma el relevo de los bloggers de moda y que ha logrado captar la atención de los profesionales de la comunicación del sector debido a su potencial en la generación de engagement con su público. El objetivo de la presente investigación es conocer el perfil de los micro fashion influencers, sus casuísticas y los protocolos de actuación que utilizan en la gestión de sus cuentas de Instagram. Para ello, se ha realizado una investigación cualitativa mediante una entrevista semiestructurada sobre una muestra seleccionada a través de la técnica no probabilística de bola de nieve. En concreto, entre mayo y junio de 2018 se realizó el trabajo de campo sobre un grupo de 10 micro instagramers de moda en España. A través de cuatro preguntas de investigación, se analiza de qué manera interactúan los micro instagramers, la relación que mantienen con las marcas y empresas de comunicación, cómo son sus publicaciones y qué motivaciones tienen para estar activos en la citada red social. Las respuestas de las micro instagramers, que acumulan una audiencia total de 240.439 seguidores, apuntan que se trata de un perfil no profesionalizado caracterizado por la ausencia de acuerdos de colaboración y una mínima remuneración económica. Además, son conscientes de sus carencias técnicas a la hora de realizar y editar sus posts.

\section{Palabras clave}

Medios sociales; Redes sociales; Instagram; Instagramers; Moda; Influenciadores; Micro influencers; Macro influencers; Engagement. 


\begin{abstract}
The evolution of social networks in general, and Instagram in particular, has led to the emergence and proliferation of digital influencers, a figure that has taken over from fashion bloggers and managed to capture the attention of communication professionals in the sector due to their potential to generate engagement with their public. The objective of the present research is to determine the profile of micro fashion influencers, their casuistics, and the protocols of action that they use when managing their Instagram accounts. For this, qualitative research was carried out through a semistructured interview of a sample selected using the nonprobabilistic snowball technique. Specifically, between May and June 2018, fieldwork was carried out with a group of ten fashion micro instagramers in Spain, analyzing four research questions regarding how micro instagramers interact with and their relationship with brands and communication companies, the nature of their publications, and their motivations for being active on the mentioned social network. The responses of the micro instagramers, with a combined audience of 240,439 followers, suggest that their nonprofessional profile is characterized by the absence of collaboration agreements and minimum economic remuneration. In addition, they are aware of their technical deficiencies when creating and editing their posts.
\end{abstract}

\title{
Keywords
}

Social media; Social networks; Instagram; Instagramers; Fashion; Influencers; Micro influencers; Macro influencers; Engagement.

\section{Introducción y estado de la cuestión}

El sector de la moda se ha visto embestido de lleno por la explosión de los nuevos medios y formatos que han provocado

"la modificación de su sistema, estructuras y estrategias; e internet se ha convertido en el canal paradigmático donde consumir información de moda inmediata, global e ilimitada” (Fernández-de-Córdova, 2017, p. 13).

La forma en la que los fashion instagramers se comunican con sus seguidores es el resultado de un proceso evolutivo que surgió con los primeros blogs sobre moda a finales del siglo XX (Findlay, 2015). A partir de 2004 estas plataformas digitales personalistas incorporaron una característica novedosa: los protagonistas hablaban de sus propios outfits, aportando valoraciones y opiniones (Rocamora; Bartlett, 2009; Findlay, 2015; Ruiz-Molina, 2013). Ese subgénero denominado blogger's personal style reflejaba también una forma de vida "real o representada" (Findlay, 2015, p. 198) donde los bloggers operaban siguiendo unas normas implícitas: las de la blogosfera (Pedroni, 2015). Los públicos se han convertido en generadores de información y contenido y

"las redes sociales facilitan el intercambio, la viralización y la amplificación de esa información" (Fernández-de-Córdova, 2017, p. 17).

Como resultado de este proceso continuado de modernización del marketing y la comunicación ha nacido un consumidor

"enormemente empoderado con un papel activo que quiere fijar relaciones con las marcas a través del diálogo transparente y fluido, en el que no le presionen" (De-Aguilera-Moyano; Baños-González; Ramírez-Perdiguero, 2016, p. 28).

En el contexto de la moda, se ha realizado especial hincapié en el "poder prescriptivo que tienen determinados grupos" (Fernández-de-Córdova, 2017, p. 30) y el alto grado de notoriedad que generan en torno a una marca o un producto (Augure, 2015; Launchmetrics, 2019). Así pues, gracias a las redes sociales

"se crean y fluyen las tendencias de manera más democrática y a una velocidad de vértigo" (Pérez-Curiel; Clavijo-Ferreira, 2017, p. 230)

con

"una tipología de usuarios y consumidores que conlleva un cambio visceral en el comportamiento de compra de moda online" (Pérez-Curiel; Luque-Ortiz, 2017, pp. 255-281).

Con respecto a los contenidos que comparten marcas, influencers y consumidores, la llegada de la Web de la imagen (Orihuela, 2015) y, más concretamente, de redes sociales como Instagram y Pinterest han convertido la Red en un lugar de intercambio de imágenes y piezas audiovisuales. De hecho, se espera que los vídeos online acaparen el 80\% de todo el tráfico de internet en 2019 (Cisco, 2015).
La forma en la que los fashion instagramers se comunican con sus seguidores es el resultado de un proceso evolutivo que surgió con los primeros blogs sobre moda a finales del siglo $X X$

\subsection{Rasgos del influenciador digital}

Los influencers tienen una característica común: comparten información que logra influir en su público. Su opinión tiñe los contenidos y

"la voz subjetiva se convierte en el foco de interés del medio digital. A través de un tono coloquial en las conversaciones se promueve la empatía con el otro, facilitada por una conexión a nivel personal y emocional" (Fernández-de-Córdova, 2017, p. 102). 
Esta conexión personalista resulta clave para obtener más seguidores en las redes sociales y alcanzar la denominada "autenticidad en el engagement" (Pérez-Curiel; Luque-Ortiz, 2017, p. 262). A pesar de que algunas marcas acuden a estrellas que tienen millones de fans en sus perfiles sociales, los expertos coinciden en que la interacción es mucho más importante que el número de espectadores (Adecec, 2016), ya que

"a veces es preferible tener una comunidad no tan grande, pero con followers fieles y constantes" (Pérez-Curiel; Luque-Ortiz, 2017, p. 262).

Es importante resaltar que algunos autores han definido el papel de las mujeres influencers en Instagram como un "having it all" (Duffy; Hund, 2015, p. 2), un concepto que hace referencia a cómo las bloggers que empezaron con pocos recursos se han convertido en mujeres exitosas e independientes económicamente. Esta consecuencia se repite como patrón en el mundo influencer debido sobre todo a tres factores:

"el destino del trabajo apasionado, la puesta en escena del glamour y el intercambio social cuidadosamente seleccionado" (Duffy; Hund, 2015, p. 4).

Así pues, los micro instagramers han evolucionado hacia el concepto de self-branding (Hearn, 2008) mediante una "práctica social que puede ser construida y aprendida" (Marwick; Boyd, 2010, p. 127). Una de las diferencias que encontramos es que hoy en día el usuario de internet puede acceder a comprar los productos de moda que llevan los bloggers directamente a través de las redes sociales, como 21 buttons o Instagram. Este signo muestra una de las tendencias que han detectado los investigadores: muchas influencers con miles de seguidores no cuentan con blog y triunfan igualmente (Pedroni, 2016; Antevenio, 2018). De hecho, Pedroni (2016) afirma que el blog

"ya no es la única plataforma de publicación de contenido, y a menudo, ni siquiera es la principal; más bien se ha convertido en el centro de una estrategia de redes sociales en la que los canales iconográficos, especialmente Instagram, toman el control" (Pedroni, 2016).

Según la opinión de los profesionales de comunicación de los sectores de moda, lujo y cosméticos (Launchmetrics, 2019), Instagram es el canal preferido para implementar campañas con influencers, con el 46,2\%, seguido de Facebook y YouTube, con $15,7 \%$ y $14,1 \%$, respectivamente; mientras que sólo el $9,3 \%$ de los responsables de marketing eligen los blogs para ello.

Con respecto a sus motivaciones profesionales, estudios sectoriales recientes señalan que poder vivir de ello y profesionalizarse es la principal meta de quienes quieren dedicarse a este sector, especialmente los millennials y los miembros de la Generación Z (Social Publi, 2018). No obstante, sólo el 47,3\% de los influencers de los sectores moda, lujo y belleza firman siempre acuerdos de colaboración con las marcas (Launchmetrics, 2019). También resulta de interés el hecho de que la motivación económica de los fashion influencers pasa del $57 \%$ al $27 \%$ de 2017 a 2018 ; mientras que en 2019 crece hasta el 69,4\% (Launchmetrics, 2017; 2018; 2019). El rol que tienen los influenciadores digitales pasa

Instagram es el canal preferido para implementar campañas con influencers de semi profesionales a profesionales; es decir, de amantes de la moda a trabajadores de un campo que incluye tanto el aspecto económico como el de la producción cultural (Pedroni; Sádaba; San-Miguel, 2017). Otros estudios recientes (Audrezet; De-Kerviler; Moulard, 2018) concluyen que los influencers se fijan objetivos económicos, aunque partiendo de la autenticidad, la pasión por la moda y sobre todo la transparencia; lo que incluye la forma en la que hacen saber a sus seguidores qué contenido es pagado por una marca y cuál no.

\subsection{Número de seguidores vs engagement}

Un requisito fundamental para lograr engagement entre marca y usuarios es conseguir interacción entre ambos a través de contenidos honestos, transparentes y sinceros (Del-Pino-Romero; Castelló-Martínez, 2017; Castelló-Martínez, 2018). La falta de esta relación directa de los macro influencers con sus seguidores hace que estos perfiles pierdan credibilidad y favorezcan la tendencia a poner en valor la figura de los micro influencers (Launchmetrics, 2018). Este perfil hace referencia a usuarios de redes sociales que

"puede que no sean tan conocidos ni tengan tantos seguidores como una celebrity, pero que, por sus actividades, intereses y/u opiniones tienen un perfil de seguidores muy bien definido y segmentado" (Del-Pino-Romero; Castelló-Martínez, 2017, p. 296).

Diferenciar los términos macro y micro influencers resulta casi imposible desde un punto de vista cuantitativo. Las cifras establecidas por la bibliografía académica fijan el límite en los 150.000 seguidores (Pérez-Curiel; Luque-Ortiz, 2017; Pérez-Curiel; Clavijo-Ferreira, 2017), mientras que los criterios de las fuentes profesionales son más dispares y consideran como micro a aquel que tiene entre 500 y 10.000 seguidores (Socialbakers, 2019; Social Publi, 2018; Kasitoko, 2017; Gilbert, 2018; Hatton, 2018); hasta 100.000 (H2H Agencia, 2017; Launchmetrics, 2019; Wolfson, 2017; García, 2018) o incluso hasta 150.000 (Boyd, 2016).

A pesar de ello, sí que existen argumentos similares a la hora de definirlos. Los micro influencers son gente real que tiene cuentas con contenido específico (Del-Pino-Romero; Castelló-Martínez, 2017), lo que permite a las marcas identificar y crear mayores tasas de engagement con sus audiencias, particularmente con los millennials (Wolfson, 2017). Aunque existen opiniones contrarias al respecto, Van-Dijck afirma que 
"cuantos más contactos tengas y hagas, más valioso te volverás, porque más personas piensan que eres popular y, por lo tanto, quieren conectar contigo" (Van-Dijck, 2013, p. 13).

En contraposición, se cree que los influencers que tienen menos fans son más auténticos y sus seguidores no cuestionan sus fotos, todo lo contrario que las grandes celebridades; son más económicos a la hora de contratar que los macro influencers, y participan en campañas fáciles de ejecutar y a través de redes sociales gratuitas.

Por otra parte, los micro influencers son los individuos que se encuentran más cerca del consumidor en el momento en el que toma la decisión de compra (Brown; Fiorella, 2013). Son gente normal que interactúa con otros de forma natural. La gran ventaja es que los micro influencers con menos de 1.000 seguidores consiguen tasas de interacción cercanas al 15\%, por lo que su capital está en la segmentación con perfiles muy especializados (Díaz-Soloaga, 2018). Por contra, no logran la repercusión que tienen los macro, también denominados meso-celebrities (Pedroni, 2016), en cuanto a número de seguidores y el alcance de sus posts, no se les puede controlar tanto y el retorno de inversión es difícil de medir (Hatton, 2018). Ante este escenario, la micro influencia se vuelve clave para alcanzar audiencias muy segmentadas. El $45,6 \%$ de los profesionales de la comunicación del sector de la moda internacional prefiere trabajar con micro y el 33,6\% opta por los macro (Launchmetrics, 2018). En esta misma línea, se ha demostrado que la fuente principal a la que acuden los millennials para informarse sobre moda es el boca a boca, o lo que se denominan

"fuentes personales, a través de conversaciones con sus círculos cercanos y fijándose en lo que lleva su entorno" (San-Miguel; Sádaba, 2019, p. 205).

Es preciso analizar la diferencia entre el boca a boca o word of mouth (WOM en sus siglas en inglés), y el boca a boca en el contexto electrónico (eWOM). La diferencia fundamental es que en el tradicional el mensaje suele ser efímero, mientras que en el eWOM se mantiene gracias al entorno digital (Cosenza; Solomon; Kwon, 2015), por lo que un usuario puede acudir en búsqueda de información tiempo después de que alguien haya dado su opinión, por ejemplo, en un foro o una red social.

\subsection{Proyección del sector}

Los últimos datos publicados por IAB Spain (2019) indican que el 68\% de los usuarios sigue a influencers a través de las redes sociales, cuatro puntos menos que el año anterior. No obstante, en el caso particular de Instagram existe un aumento del 5\%, del 35\% al 40\%, con especial incidencia en el rango de edad de 16 a 45 años. Conscientes de este potencial, las marcas centran sus esfuerzos en dirigir sus comunicaciones a líderes de opinión, o fashion influentials (San-Miguel; Sádaba, 2019), en lugar de hacerlo directamente al público objetivo final (Hall, 2016). Y es que para los consumidores la publicidad más fiable de un producto o servicio no es otra que las recomendaciones de sus familiares y amigos. En concreto, tres de cada cuatro confieren mayor credibilidad a la experiencia de las personas de su entorno. Además, un $55 \%$ se fía de los comentarios que otros consumidores postean (Nielsen, 2015).

En datos económicos, el presupuesto dedicado a las campañas de influencers creció globalmente en 2017 entre un $3 \%$ y un $6 \%$; el $48,2 \%$ de los profesionales del sector moda destinó entre un $10 \%$ y un $40 \%$ del montante a este tipo de estrategias (Launchmetrics, 2018); y el 48,3\% invierte más de 10.000 euros al año. El presupuesto destinado a influencer marketing continuará creciendo, según el 60\% de los profesionales de la industria (Launchmetrics, 2019). Por su parte, un 58\% de los profesionales de la comunicación ha contratado en 2019 los servicios en redes de influencers, principalmente en Instagram, un dato que aumenta en $12 \%$ con respecto a 2018; y además el $87 \%$ de ellos está bastante o muy satisfecho (IAB Spain, 2018; 2019). De hecho, el 93\% de los profesionales de la comunicación asevera que las relaciones con influencers son una fórmula más eficaz que la promoción y la publicidad tradicional para aumentar la visibilidad y el valor de marca (Augure, 2015; Adecec, 2016).

\section{Material y métodos}

El objetivo principal de la investigación es definir el perfil de los micro instagramers en España a través del análisis de sus casuísticas y protocolos de actuación. Para ello se plantean cuatro preguntas de investigación:

1. ¿¿De qué manera actúan y difunden sus publicaciones estos perfiles en Instagram?

2. ¿Cómo es su relación con las marcas/empresas de comunicación?

3. ¿Cómo son sus publicaciones en Instagram?

4. ¿Qué motivaciones tienen para estar en esta red social?

En concreto, se ha llevado a cabo una metodología cualitativa basada en la realización de entrevistas semiestructuradas a micro instagramers de moda españoles, entre mayo y junio de 2018. La elección de la muestra se realizó a través de un muestreo no probabilístico mediante un proceso de difusión por bola de nieve exponencial (Ochoa, 2015). Se ha optado por una muestra denominada de "intención estratégica" con el fin de recabar información de aquellas personas que "pueden o quieren hacerlo" (Cea-D’Ancona, 2004, p. 171). En muestras muy pequeñas, de menos de 30 individuos, como es el caso de este estudio sobre influencers de moda en España, 
"el muestreo estratégico puede favorecer la consecución de información más relevante que la obtenida de una muestra del mismo tamaño extraída al azar de la población de estudio" (Cea-D’Ancona, 2004, p. 171).

Tal y como se refleja en la tabla 1 , la investigación se realiza sobre un total de $10 \mathrm{mi}-$ cro instagramers seleccionados a partir del criterio del número de seguidores establecido en menos de 150.000 (Pérez-Curiel; Luque-Ortiz, 2017; Pérez-Curiel; Clavijo-Ferreira, 2017).

Como se puede observar, atendiendo a la catalogación realizada por Posner (2016), seis de los micro instagramers pertenecen a la Generación Y o millennials (nacidos entre 1982 y 2002). Se trata de una generación que ha crecido con la tecnología, están más formados y viven su vida a través de internet. Otros tres micro instagramers están dentro de la Generación Z (nacidos entre 1995 a 2010), estos son los nativos digitales que más redes sociales usan a la vez (IAB Spain, 2018). Por último, encontramos un perfil que no forma parte de la Generación X (nacido entre 1961 y 1981), y a esta generación se la define como perteneciente al grupo de los desencantados que se han convertido en yupsters.

Por otro lado, y con la finalidad de obtener una mayor aproximación y conocimiento del perfil de los entrevistados, se ha realizado un análisis de los posts publicados por los micro instagramers entre el 1 de mayo y el 30 de junio de 2018. Para ello se parte de los protocolos utilizados previamente por Pérez-Curiel y Sanz-Marcos (2019). De esta manera, en un primer bloque relativo a la métrica se contabilizan los posts publicados, así como los "me gusta" y "comentarios" de los usuarios (totales y media por post) (tabla 2). En un segundo bloque se analiza el contenido de los posts, desde el etiquetado y referencias a la marca, las alusiones promocionales (descuentos, sorteos y publicidad), los recursos audiovisuales (vídeo, foto o imagen) y los recursos de interacción (enlaces, hashtags y menciones) (tabla 3).

Finalmente, a partir de dicha muestra se planteó una entrevista semiestructurada para responder a las preguntas de investigación planteadas. En la tabla 4 se detallan los temas y aspectos analizados.

\section{Análisis y resultados}

Los resultados de la investigación se presentan en cuatro bloques que atienden a los cuatro objetivos planteados.

\subsection{Manera de interactuar en Instagram}

Respecto a su forma de utilizar Instagram, no hay ningún micro instagramer que dedique la jornada en exclusivo a gestionar su cuenta de Instagram: seis de ellos invierten entre 6 y 15 horas, mientras que tres lo hacen menos de 5 horas y el último no aporta esta información. Asimismo, si atendemos a los años que llevan trabajando en su perfil, la mitad afirma que su cuenta en Instagram tiene una antigüedad de entre 1-2 años; otros tres, entre 3-5 años; y los dos restantes, desde 6 ó más. Además, todos los entrevistados tienen el perfil público y detallan que lo que comparten no se publica directamente en Facebook, tal y como permite la aplicación. Todos aseguran que Instagram es su plataforma principal y sólo uno usa Facebook con igual intensidad. Además de la citada red social, siete de los 10 entrevistados también utilizan Facebook y tres tienen blog. 


\subsection{Relación con las marcas/empresas de comunicación}

Los datos obtenidos reflejan que cuatro de los influenciadores participan en una campaña publicitaria al mes y otros cuatro, entre 2 y 5 campañas. Tan sólo uno afirma participar en 11 o más campañas. Por otra parte, se observa cómo la periodicidad con la que comparten fotos sobre una marca con la que tienen un acuerdo varía según el entrevistado. Cuatro afirman que depende de diversos factores y, principalmente, de las decisiones de las marcas; dos de los micro influencers publican contenido patrocinado una vez a la semana; otros dos, cada 15 días; otro, una vez al mes; y el restante, varios días a la semana.

Asimismo, todos coinciden en que el correo electrónico es el principal medio a través del cual las marcas o empresas de comunicación se ponen en contacto con ellos. Otro de los medios destacados por ocho de los entrevistados son los mensajes privados de Instagram, y tan sólo uno ha sido contactado alguna vez través de mensajes públicos en un post.

Tabla 3. Análisis del contenido de los posts publicados por los entrevistados. Fuente: Instagram (1 mayo a 30 junio 2018)

\begin{tabular}{|c|c|c|c|c|c|c|c|c|c|}
\hline \multirow[b]{3}{*}{$\begin{array}{l}\text { Micro ins- } \\
\text { tagramer } \\
\text { entrevis- } \\
\text { tado }\end{array}$} & \multicolumn{9}{|c|}{ Bloque II } \\
\hline & \multicolumn{9}{|c|}{ Contenido de los posts (\%) } \\
\hline & $\begin{array}{c}\text { Etiqueta la } \\
\text { marca }\end{array}$ & $\begin{array}{l}\text { Hace referen- } \\
\text { cia al producto } \\
\text { que muestra }\end{array}$ & $\begin{array}{l}\text { Menciona } \\
\text { un concurso, } \\
\text { sorteo o des- } \\
\text { cuento }\end{array}$ & $\begin{array}{l}\text { Incluye } \\
\text { enlace a } \\
\text { marca }\end{array}$ & $\begin{array}{l}\text { Incluye } \\
\text { fotos }\end{array}$ & $\begin{array}{c}\text { Incluye } \\
\text { imágenes } \\
\text { (carteles, } \\
\text { dibujos...) }\end{array}$ & $\begin{array}{l}\text { Incluye } \\
\text { vídeos }\end{array}$ & $\begin{array}{c}\text { Incluye } \\
\text { hashtags }\end{array}$ & $\begin{array}{c}\text { Incluye } \\
\text { menciones }\end{array}$ \\
\hline 1 & 100 & 21,9 & 12,5 & 6,3 & 100 & 0,0 & 0,0 & 0,0 & 0,0 \\
\hline 2 & 13,8 & 3,4 & 6,9 & 0,0 & 100 & 0,0 & 0,0 & 0,0 & 0,0 \\
\hline 3 & 76,9 & 7,70 & 0,0 & 0,0 & 100 & 0,0 & 0,0 & 0,0 & 0,0 \\
\hline 4 & 21,0 & 11,4 & 4,5 & 0,0 & 90,9 & 6,8 & 2,3 & 2,3 & 0,0 \\
\hline 5 & 76,9 & 7,7 & 0,0 & 0,0 & 100 & 0,0 & 7,7 & 0,0 & 0,0 \\
\hline 6 & 47,4 & 0,0 & 26,3 & 0,0 & 100 & 0,0 & 0,0 & 0,0 & 0,0 \\
\hline 7 & 83,3 & 0,0 & 0,0 & 0,0 & 100 & 0,0 & 0,0 & 0,0 & 0,0 \\
\hline 8 & 42,9 & 5,2 & 1,3 & 1,3 & 100 & 0,0 & 0,0 & 0,0 & 0,0 \\
\hline 9 & 50,0 & 0,0 & 2,9 & 0,0 & 100 & 0,0 & 0,0 & 0,0 & 0,0 \\
\hline 10 & 35,7 & 0,0 & 7,1 & 0,0 & 100 & 0,0 & 0,0 & 0,0 & 0,0 \\
\hline
\end{tabular}

Tabla 4. Variables a analizar en función de las preguntas de investigación

\begin{tabular}{|c|c|}
\hline Preguntas de investigación & Aspectos a analizar \\
\hline $\begin{array}{l}\text { ¿De qué manera actúan y difunden sus } \\
\text { contenidos en Instagram? }\end{array}$ & $\begin{array}{l}\text { Asiduidad con la que publican posts. } \\
\text { Tiempo que le dedican. } \\
\text { Años que llevan con su perfil activo. } \\
\text { Si es su red social principal. } \\
\text { Desde qué dispositivos editan sus fotografías. }\end{array}$ \\
\hline $\begin{array}{l}\text { ¿Cómo es su relación con las marcas/ } \\
\text { empresas de comunicación? }\end{array}$ & $\begin{array}{l}\text { En cuántas campañas publicitarias participan. } \\
\text { Periodicidad con la que publican las fotos de una marca con la que han llegado a un acuerdo. } \\
\text { Medios a través de los cuáles las empresas se ponen en contacto y quién se encarga. } \\
\text { Si les piden informes previos al inicio de campaña. } \\
\text { Si reciben regalos. } \\
\text { Si pueden elegir el producto que publicitan. } \\
\text { Si firman algún contrato y las condiciones que les imponen. } \\
\text { Conocer qué es lo que creen que más valoran las agencias de comunicación de su perfil. } \\
\text { Qué tipo de productos o marcas promocionan. }\end{array}$ \\
\hline $\begin{array}{l}\text { ¿Cómo son sus publicaciones en } \\
\text { Instagram? }\end{array}$ & $\begin{array}{l}\text { Perfil que asumen a la hora de publicar contenido. } \\
\text { Qué se ve en su perfil. } \\
\text { De qué calidad consideran sus posts. } \\
\text { Las características de sus fotos; } \\
\text { Las diferencias que hay entre las publicaciones patrocinadas y las que no lo son. } \\
\text { Sobre las imágenes de productos patrocinados se busca conocer si tienen libertad artística y si } \\
\text { indican que se trata de contenido publicitario. } \\
\text { Si aprovechan todos los recursos que ofrece Instagram. }\end{array}$ \\
\hline $\begin{array}{l}\text { ¿Qué motivaciones tienen para estar en } \\
\text { esta red social? }\end{array}$ & $\begin{array}{l}\text { Por qué están en Instagram. } \\
\text { Si supone un trabajo o un hobby. } \\
\text { Lo que obtienen personalmente al colaborar con marcas. } \\
\text { Si han notado un incremento de followers durante el último año. } \\
\text { Si son conscientes de la compra de seguidores. } \\
\text { Si conocen la diferencia entre macro y micro influencers. } \\
\text { Si reciben compensación económica. }\end{array}$ \\
\hline
\end{tabular}


Por su parte, todos los micro instagramers de la muestra afirman que son las agencias las que se suelen poner en contacto con ellos, aunque hay tres que también reciben comunicaciones por parte de los departamentos de marketing y comunicación de las marcas. Y tan sólo tres de los entrevistados mencionan a las agencias especializadas en influencer marketing como interlocutoras. En cualquier caso, siete de los micro influencers confirman que no se les piden informes antes de proponerles una acción con una marca, y tan sólo la mitad asegura que firma contratos de colaboración.

Por otro lado, todos aseguran que reciben siempre o casi siempre experiencias o productos gratuitos y que se sienten libres a la hora de elegir y seleccionar lo que van a publicitar en sus posts. La totalidad de los entrevistados etiquetan a marcas que no se lo han pedido y aseguran que nunca les han hecho saber que no quieren que las etiqueten. Sin embargo, cuando existe una colaboración, los micro instagramers afirman recibir instrucciones sobre cómo debe ser la creatividad o el contenido de las publicaciones. Otras condiciones impuestas por las marcas son:

- que se etiquete a la marca;

- que se publiquen en una fecha determinada;

- que sigan unos criterios de imagen determinados;

- que la foto sea de calidad y que se identifique bien el producto o marca;

- que incorporen hashtags y menciones de la promoción o marca;

- que se cumplan unos horarios determinados.

Los entrevistados creen que el número de seguidores, la calidad y la originalidad de las fotografías son los aspectos que más valoran las marcas para seleccionarlos. Seguidos de estos y por orden de importancia se encuentran la cantidad de likes, la repercusión que tienen los posts y la afinidad del influencer con el producto. Por contra, consideran que lo menos valorado es el uso de hashtags y el lugar de residencia.

En cuanto a los productos más promocionados, la ropa y los complementos, seguidos de la joyería y la relojería, son los que más publicitan (tabla 5).

Tabla 5. Temas de los posts y marcas más etiquetadas

\begin{tabular}{|c|c|c|}
\hline $\begin{array}{l}\text { Micro instagramer } \\
\text { entrevistado }\end{array}$ & Tema de los posts & Marcas que etiquetan con frecuencia \\
\hline 1 & Moda de calle | Viajes | Moda elitista | Festivales & $\begin{array}{l}\text { Zara } \mid \text { Bershka } \mid \text { Chanel | Louis Vuitton | Mango | Gadeashoes | Dior | } \\
\text { Yamamay }\end{array}$ \\
\hline 2 & $\begin{array}{l}\text { Moda de calle | Viajes | Fiestas con amigos | } \\
\text { Festivales }\end{array}$ & Zara |Sandroparis | Anandlee | Woonivers | Daniel Wellington \\
\hline 3 & $\begin{array}{l}\text { Moda de calle | Viajes | Fiestas con amigos | Moda } \\
\text { elitista }\end{array}$ & Massimo Dutti| Dior |Rayban |Zara |Mango|Sandro Paris \\
\hline 4 & Moda de calle | Viajes & Aussie Hair | Maybelline | Dior | Rental Mode | \\
\hline 5 & Moda de calle | Viajes & Zara $\mid$ Mango |Robin Collection |Pantene \\
\hline 6 & $\begin{array}{l}\text { Moda de calle | Viajes | Moda elitista | Asesora- } \\
\text { miento de moda | Fotografías creativas | Festivales }\end{array}$ & Daniel Wellington | Cluse | Tijneyewear \\
\hline 7 & Moda de calle & Zara|21_buttons|María Pascual \\
\hline 8 & $\begin{array}{l}\text { Moda de calle | Viajes | Asesoramiento de moda | } \\
\text { Fotografías creativas }\end{array}$ & Conforama | Granier | Paul Hewitt | Riadz | Galenic| Bershka \\
\hline 9 & $\begin{array}{l}\text { Moda de calle | Moda elitista | Asesoramiento de } \\
\text { moda }\end{array}$ & 21_buttons |Zara |Dior|Chanel \\
\hline 10 & Moda de calle / viajes & Zara|Uterque Oficial|21_buttons \\
\hline
\end{tabular}

En contraposición, los productos de alimentación son los que menos les ofrecen. Asimismo, los resultados reflejan que entre el $70 \%$ y el $80 \%$ de las llamadas que reciben los influencers que tienen entre 50.001 y 100.000 fans son de marcas internacionales; mientras que la media de contactos de firmas internacionales baja hasta el 48,57\% en el caso de aquellos que tienen entre 10.001 y 50.000 fans (siete individuos de la muestra). La única persona que tiene entre 5.001 y 10.000 seguidores no recibe ninguna comunicación por parte de marcas muy conocidas.

\subsection{Relativo a sus publicaciones}

El perfil mayoritario que asumen los micro instagramers en los posts patrocinados es el de cliente, en siete de los casos. Además:

- la mitad de los entrevistados afirma actuar como espacio publicitario;

- como fan, cuatro influencers;

- como modelo, tres;

- como persona anónima, en sólo uno de los casos. 
Cabe resaltar que todos afirman que etiquetan a la marca casi siempre para hacer saber que un post es publicitario y corresponde a una firma en particular. Entre las temáticas o contextualizaciones de las publicaciones, tal y como se refleja en el gráfico 1 , destacan la moda de calle y los viajes (imagen 1 ).

Por otra parte, respecto a las fotos publicadas:

- la mitad de los entrevistados piensa que son de una calidad media;

- tres de los participantes, que son buenas;

- dos, muy buenas.

Cabe destacar que:

- nueve de los 10 contactados aseguran que capturan sus fotos con el teléfono móvil;

- cuatro también lo hacen con una cámara profesional;

- otros dos, con una compacta.

Además, se confirma que todos los entrevistados editan las fotos que suben a Instagram, aunque mayoritariamente lo hacen con una aplicación del teléfono móvil. Tan sólo uno de los micro instagramers afirma utilizar un programa de ordenador.

Las principales diferencias que los entrevistados encuentran entre las publicaciones personales y las patrocinadas se centran en que para estas últimas suele haber un uso de hashtags concretos. A este respecto, la micro instagramer 6 añade que:

"aunque depende del producto que sea, en general le doy siempre mi toque personal".

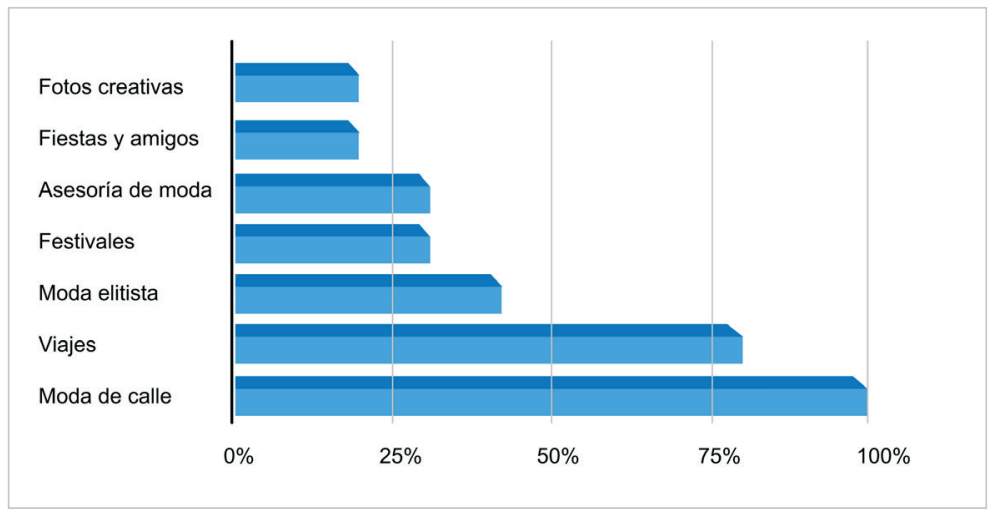

Gráfico 1. Contenido de su Instagram
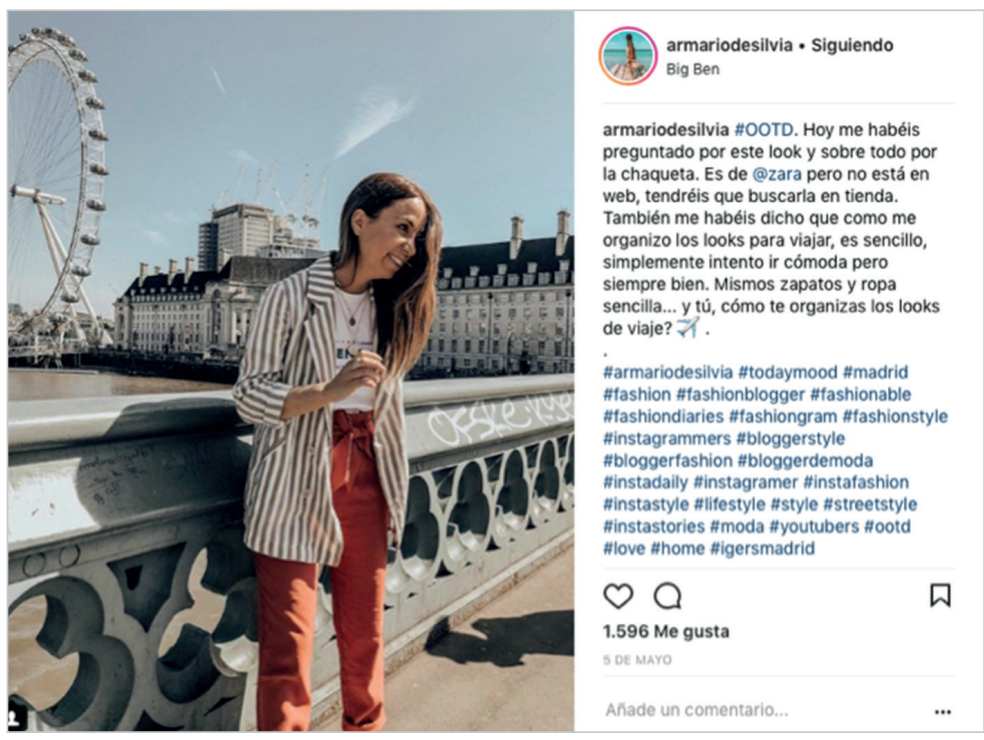

Imagen 1. Ejemplo de post de viajes

Fuente: Instagram, 5 de mayo de 2018

Por otro lado, afirman que los criterios que siguen a la hora de aceptar realizar una publicación patrocinada son principalmente de afinidad con el producto:

"que la marca se adapte a mi estilo y me identifique" (Micro instagramer 1);

"que sea una marca que yo usaría" (MI 3);

"que me identifique" (MI 4);

"que me haga ser yo misma" (MI 5);

"que lo que me ofrezcan sea de mi estilo o me guste" (MI 7);

"sigo el criterio que fija la marca sin perder mi esencia" (MI 10).

El etiquetado de las piezas no es una práctica que siempre realizan. Mientras que ocho de los entrevistados constatan que etiquetan siempre a la marca, dos de ellos aseguran que no lo hacen siempre. Los datos son distintos a la hora de indicar si el contenido es patrocinado, mientras que más de la mitad de ellos afirma indicarlo con asiduidad, cuatro micro instagramers contestan que casi nunca lo reflejan. De forma mayoritaria, nueve de los diez participantes exponen que etiquetan a la marca para indicar que se trata de una foto patrocinada y seis de ellos usa hashtags como \#publicidad \#ad \#advertising para tal fin (imagen 2).
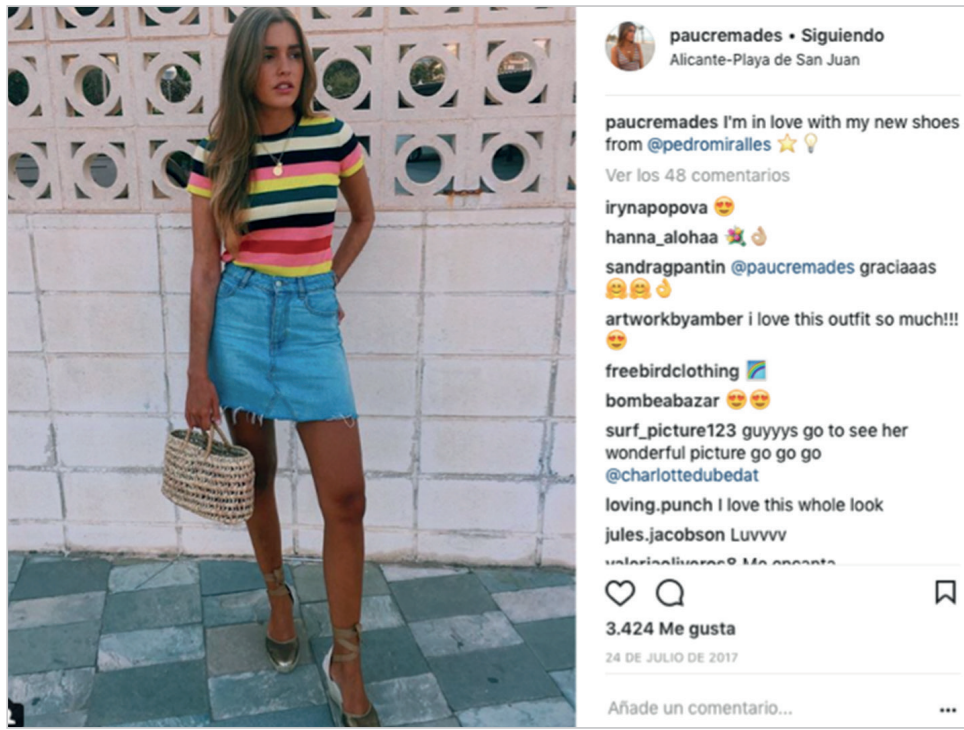

Imagen 2. Ejemplo de post de moda en el que se realiza el sorteo de una prenda de ropa y se etiqueta a la marca @dustandsouldofficial.

Fuente: Instagram, 1 de mayo de 2018 
En lo que respecta a la libertad artística, la gran mayoría, un total de nueve participantes, tienen siempre o casi siempre poder de decisión sobre sus publicaciones. Tres aseguran ser siempre ellos los que tienen la libertad artística de sus fotografías y sus posts. Coinciden todos al asegurar que realizan las fotos pensando en gustar a sus seguidores. En lo referente a su audiencia, seis de los influenciadores piensan en gustar a sus fans. Por último, seis indican siempre o casi siempre que el contenido es patrocinado y los otros cuatro contestan que casi nunca lo reflejan. De forma mayoritaria, nueve de los diez participantes exponen que etiquetan a la marca para indicar que se trata de una foto patrocinada y seis de ellos usa hashtags como \#publicidad \#ad \#advertising para tal fin.

Por último, todos los micro influencers entrevistados suben fotos propias a Instagram y ocho usa las stories, que tienen una duración de 24 horas. Por contra, los vídeos e Instagram direct son usados en muy pocas ocasiones.

\subsection{Motivación del influencer}

Prácticamente todos los micro instagramers que conforman la muestra utilizan esta red social porque les gusta la moda. En concreto, tres de los micro instagramers afirman sentir "amor a la moda", mientras que las motivaciones de otros son: "me encanta estar en Instagram" o "conseguir seguidores y crear mi marca personal".

La mayoría de los entrevistados valora positivamente el hacer networking con personas relevantes como una de las razones

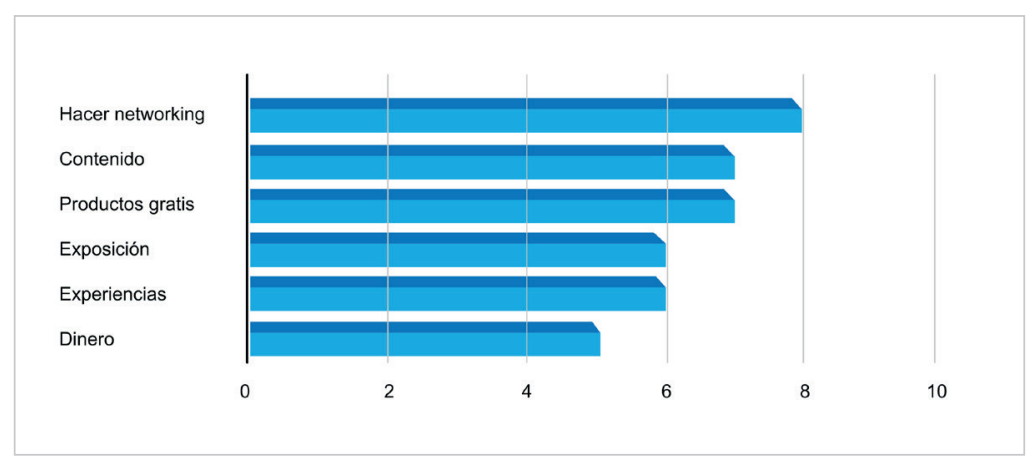

Gráfico 2. Lo que obtienen al colaborar con marcas principales para colaborar con marcas, mientras que siete de ellos se refieren a la obtención de productos y contenido de valor para compartir. Por contra, la compensación económica resulta lo menos importante (gráfico 2).

Siete de los 10 entrevistados tienen a 15 de junio del 2018 entre 10.001 y 50.000 followers, a los que les siguen los dos que cuentan con entre 50.001 y 100.000 y el único que está entre los de 5.001 a 10.000 seguidores. Además, en el último año:

- cuatro han sumado entre 5.001 y 10.000 followers;

- otros cuatro, entre 1.001 y 5.000 ;

- uno, entre 10.001 y 50.000 ;

- el restante, entre 100 y 1.000.

Comparando estos datos con el número de fans, sólo un influenciador ha conseguido un incremento de entre 10.001 y 50.000 usuarios, y es el que tiene entre 50.001 y 100.000 .

Tabla 6. Relación de los micro instagramers con las marcas

\begin{tabular}{|c|c|c|c|l|c|}
\hline $\begin{array}{c}\text { Micro instagramer } \\
\text { entrevistado }\end{array}$ & Seguidores & Edad & $\begin{array}{c}\text { Recibe regalos } \\
\text { de las marcas }\end{array}$ & $\begin{array}{c}\text { Percibe remuneración } \\
\text { económica por parte de las marcas }\end{array}$ \\
\hline 1 & 22.000 & 29 & Si & Menos de 1.000 euros al año \\
\hline 2 & 10.900 & 26 & Si & No & No \\
\hline 3 & 7.600 & 34 & Si & Menos de 1.000 euros al año \\
\hline 4 & 13.000 & 27 & Si & No \\
\hline 5 & 27.000 & 22 & SI & Menos de 1.000 euros al año \\
\hline 6 & 13.000 & 23 & Si & Menos de 1.000 euros al año \\
\hline 7 & 23.700 & 23 & Si & Menos de 1.000 euros al año \\
\hline 8 & 62.300 & 40 & Si & Entre 5.000 y 20.000 euros al año \\
\hline 9 & 50.100 & 22 & Si & No contesta \\
\hline 10 & 10.839 & 18 & Si & Entre 5.000 y 20.000 euros al año & Si \\
\hline
\end{tabular}

Si comparamos el número de seguidores con la cantidad monetaria que consiguen, vemos cómo los que no perciben ingresos tienen entre 10.001 y 50.000 seguidores; y de los que facturan entre 5.000 y 20.000 euros, la mitad tiene entre 10.001 y 50.000 seguidores y la otra mitad entre 50.001 y 100.000 fans. Además, siete de los micro instagramers aseguran que han visto un incremento de regalos recibidos en el último año comparado con el anterior.

Por otra parte, atendiendo a la edad:

- cuatro de los micro instagramers tienen entre 18 y 24 años;

- tres, entre 25 y 29 ;

- uno, entre 30 y 34 ;

- uno, entre 40 y 45;

- uno, menos de 18 años. 
Si comparamos los años con el número de seguidores, se observa que no hay correlación, ya que las dos personas con mayor número de seguidores (entre 50.001 y 100.000), tienen 22 y 40 años.

En cuanto a su ocupación principal, tan sólo un entrevistado constata que ejercer de influencer es su trabajo principal, mientras que el resto estudia o trabaja, o hace las dos cosas, además de gestionar su canal de Instagram. Con respecto a lo que ingresan al año como influencers, sin contar los regalos, la mitad (cinco) recibe menos de 1.000 euros; dos reciben entre 5.000 y 20.000 euros; y los dos restantes no perciben ningún ingreso en concepto de colaboración con las marcas.

Por último, mientras que la mayoría de ellos no se consideran macro influencers, tres de los entrevistados si piensan que lo son. En cuanto a la compra de seguidores, la práctica totalidad de los entrevistados (nueve) afirma haber oído hablar de esta práctica y la mitad opina incluso reconocer que es algo habitual, aunque ninguno de ellos desvela haberlo hecho en alguna ocasión.

\section{Discusión y conclusiones}

Los resultados obtenidos en la presente investigación permiten extraer conclusiones relevantes que nos ayudan a realizar una primera aproximación al perfil de los micro instagramers en España.

La primera conclusión es que el perfil de los instagramers de menos de 100.000 seguidores del sector moda en España en 2018, o denominados micro influencers, se autopercibe como poco profesionalizado. Es decir, estos perfiles realizan una función por la que no reciben una remuneración significativa ni está sujeta a contratos. En las entrevistas se ha ratificado la idea de que la gestión de sus perfiles en Instagram no supone la ocupación principal de los micro instagramers. Pese a que, existen estudios sectoriales (Social Publi, 2018; Launchmetrics, 2019) que defienden que poder vivir de ello es el objetivo de este tipo de influenciadores, especialmente de los millennials y los miembros de la Generación Z, la compensación económica no aparece como una motivación principal para los entrevistados.

Siguiendo este hilo argumentativo, la falta de profesionalización se evidencia también en las rutinas y procedimientos de actuación. Así pues, se ha constatado que los micro instagramers de moda no tienen una relación continuada con las marcas ni entregan informes previos a las agencias. Asimismo, la mayoría de las entrevistadas usan casi de manera exclusiva la red social Instagram y ni si quiera tienen blog. En este sentido se observa cómo se cumple la tendencia que apunta Pedroni (2016) sustituyéndose la estructura tradicional de la web en formato blog por la de la propia red social Instagram. Esta realidad se constata en estudios sectoriales (Launchmetrics, 2019), que reflejan que Instagram es el canal preferido para implementar acciones con influencers, muy por encima de los blogs. De este modo cambia la forma de consumo de la información de moda a través de los micro influencers, que a pesar de tener menor número de seguidores que los macro, cuentan con miles de ellos.

Por otra parte, los micro influencers reconocen que sus conocimientos técnicos son básicos y no utilizan herramientas profesionales de edición. Aunque algunos de ellos emplean dispositivos profesionales, todos usan de manera habitual el móvil para hacer las fotos y reconocen en algunos casos que son de calidad media. De manera excepcional, tan sólo una de las micro influencers asegura utilizar programas de tratamiento y edición de fotografías. Manifiestan ser conscientes de no aprovechar todas las funciones que permite Instagram y que ampliar sus conocimientos técnicos para la elaboración de sus publicaciones, como editar vídeos, les haría más competitivos.

Como segunda conclusión se observa que los micro instagramers cumplen con los aspectos clave que pueden propiciar de forma natural un mayor engagement (Díaz-Soloaga, 2018; Wolfson, 2017). Esta idea se sustenta en tanto en cuanto aceptan para colaborar y eligen para etiquetar sólo productos que les identifican y son compatibles con sus valores; tienen libertad artística para realizar sus fotografías y, pese a tener indicaciones de las marcas relativas a cumplimiento de horarios o menciones, elaboran los posts pensando principalmente en sus seguidores. Esta forma de proceder más próxima y auténtica genera lo que Pérez-Curiel y Luque-Ortiz (2017) llaman autenticidad en el engagement y provoca que las audiencias interactúen mucho más ante un mismo mensaje difundido por un influencer de forma próxima y auténtica, que por el de una marca.

En tercer lugar, se concluye que los micro instagramers no identifican de manera habitual las colaboraciones como publicidad de manera explícita (\#ad, \#publicidad). La tendencia detectada y corroborada en el análisis de los posts es que los micro influencers publican fotos que no siempre etiquetan y, en ocasiones, etiquetan fotos con firmas que no se lo han pedido previamente y, al no especificar cuáles son publicidad y cuáles no, los consumidores no pueden saber qué contenido está pagado. En sus publicaciones, las imágenes de productos patrocinados se mezclan con las de marcas con las que no hay una colaboración. En esta línea, tan sólo etiquetan a la marca para indicar que se trata de una foto patrocinada ya que, a pesar de que afirman usar hashtags del tipo \#ad o \#publicidad, durante el período de estudio no se ha localizado ningún post con dicha etiqueta, aunque si el de \#colaboración.

\section{La gestión de sus perfiles en Instagram no supone la ocupación principal de los micro instagramers}


Por último, se corrobora que no existe unanimidad a la hora de fijar los criterios de catalogación ni definición de los términos micro y macro influencer, ni siquiera entre los propios entrevistados. Así pues, dada la relevancia y proyección del perfil, existe una necesidad de ahondar en la definición del término que incluya sus características y su relación real y contractual con las marcas.
No existe unanimidad a la hora de fijar los criterios de definición de los términos micro y macro influencer, ni siquiera entre los propios entrevistados

La principal limitación que presenta la investigación es el reducido número de entrevistados, que no permite extrapolar los resultados a todo el universo de micro instagramers de moda en España, pero que puede dar una primera aproximación para reflejar las características más habituales tanto de sus rasgos característicos como de su modus operandi, ya que se ha analizado el perfil de un grupo de 10 micro influencers que tiene, entre todos sus miembros, una audiencia de 240.439 seguidores. Además, la técnica de muestreo por bola de nieve ha provocado la falta de control sobre la constitución de la muestra y no se ha garantizado la representatividad, por lo que no se ha podido fijar, a priori, el tamaño de muestra que se iba a obtener.

Asimismo, cabe añadir que el presente análisis se ha centrado en los propios micro instagramers de moda españoles, mientras que para tener una valoración y aproximación completa del perfil, habría que estudiarlo desde otras perspectivas como los seguidores, las marcas, etc.

\section{Referencias}

Adecec (2016). Trabajar con influencers. Fórmulas para una relación eficaz.

http://www.adecec.com/pdf/adecec_presenta_la_guia_\%E2\%80\%9Ctrabajar_con_influencers.formulas_para_una_ relacion_eficaz\%E2\% $80 \% 9 D_{-} . p d f$

Antevenio (2018). "Top ten: influencers de moda en Instagram". Antevenio, 2 febrero. https://www.antevenio.com/blog/2018/02/influencers-de-moda-en-instagram

Audrezet, Alice; De-Kerviler, Gwarlann; Moulard, Julie-Guidry (2018). “Authenticity under threat: When social media influencers need to go beyond self-presentation". Journal of business research, v. 45, pp. 170-172.

https://cutt.ly/udMZq1F

https://doi.org/10.15444/GFMC2017.03.01.05

Augure (2015). Estatus y prácticas de las relaciones con influencers en 2015.

https://bit.ly/2Ln4qgc

Boyd, Sara (2016). "How Instagram micro influencers are changing your mind one sponsored post at a time”. Forbes, 28 June.

https://bit.ly/2DCjm95

Brown, Danny; Fiorella, Sam (2013). Influence marketing. Indianapolis (USA): Que publishing. ISBN: 9780789751041

Castelló-Martínez, Araceli (2018). "Campañas publicitarias exitosas y tendencias publicitarias: una relación simbiótica”. En: Creative industries global conference: Libro de actas. Universidad de Alicante, pp. 293-309. ISBN: 9788461793877 https://dialnet.unirioja.es/servlet/articulo?codigo $=6301582$

Cea D’Ancona, María-Ángeles (2004). Métodos de encuesta. Teoría y práctica, errores y mejora. Madrid: Síntesis. ISBN: 9788497562508

Cisco (2015). "Cisco visual networking index predicts IP traffic to triple from 2014-2019; Growth drivers include increasing mobile access, demand for video services". The network, 27 May.

https://bit.ly/2beouQc

Cosenza, Tracy-Ricman; Solomon, Michael R.; Kwon, Wi-Suk (2015). “Credibility in the blogosphere: A study of measurement and influence of wine blogs as an information source". Journal of consumer behaviour, v. 14, n. 2, pp. 71-91. https://doi.org/10.1002/cb.1496

De-Aguilera-Moyano, Joaquín; Baños-González, Miguel; Ramírez-Perdiguero, Francisco-Javier (2016). “Los mensajes híbridos en el marketing posmoderno: una propuesta de taxonomía". Icono 14, v. 14, n. 1, pp. 26-57.

https://doi.org/10.7195/ri14.v14i1.890

Del-Pino-Romero, Cristina; Castelló-Martínez, Araceli (2017). “La estrategia publicitaria basada en influencers. El caso de SmartGirl by Samsung”. En: Castelló-Martínez, Araceli; Del-Pino-Romero, Cristina (coords.). Publicidad y convergencia mediática. Nuevas estrategias de comunicación persuasiva. Sevilla: Egregius Ediciones, pp. 116-146. ISBN: 97884 94697869

http://rua.ua.es/dspace/handle/10045/69329 
Díaz-Soloaga, Carmen (2018). "Ejemplos de micro influencers, todo lo que necesitas saber sobre ellos". Social media pymes, 6 marzo.

https://www.socialmediapymes.com/ejemplos-de-micro influencers-estudio

Duffy, Broke-Erin; Hund, Emily (2015). "'Having it all’ on social media: Entrepreneurial femininity and self-branding among fashion bloggers". Social media+ society, v. 1, n. 2, pp. 1-5.

https://doi.org/10.1177/2056305115604337

Establés, María-José; Guerrero-Pico, Mar; Contreras-Espinosa, Ruth S. (2019). "Jugadores, escritores e influencers en redes sociales: procesos de profesionalización entre adolescentes". Revista latina de comunicación social, n. 74, pp. $214-236$. https://doi.org/10.4185/RLCS-2019-1328

Fernández-de-Córdova, Isabel (2017). Comunicar la moda en internet: medios, marcas, influencias y usuarios. Barcelona: Editorial UOC. ISBN: 9788491168591

Findlay, Rosie (2015). "The short, passionate, and close-knit history of personal style blogs". Fashion theory, v. 19, n. 2, pp. 157-178.

https://doi.org/10.2752/175174115X14168357992319

García, Óscar (2018). “Micro influencers: ¿Cómo trabajar con ellos?”. Lift improving business, 30 mayo. https://bit.ly/2DCkhGz

Gilbert, Susan (2018). "Know the difference between micro and macro influencer marketing". Personal branding blog, 27 April.

https://www.personalbrandingblog.com/know-difference-micro-macro-influencer-marketing

Hall, John (2016). "The influencer marketing gold rush is coming: Are you prepared?”. Forbes, 17 April. https://bit.ly/2ZFoOwk

Hatton, Georgia (2018). Micro influencers vs macro influencers. Social media today, 13 February. https://bit.ly/2Gt5vkz

Hearn, Alison (2008). "Meat, mask, burden: Probing the contours of the branded 'self'". Journal of consumer culture, v. 8, n. 2, pp. 197-217.

https://bit.ly/2K3ixec

https://doi.org/10.1177/1469540508090086

H2H Agencia (2017). "El fraude en la publicidad en Instagram". H2H, 12 julio. https://humantohuman.es/el-fraude-de-la-publicidad-en-instagram

IAB Spain (2018). Estudio anual de redes sociales 2018.

https://iabspain.es/estudio/estudio-anual-de-redes-sociales-2018

IAB Spain (2019). Estudio anual de redes sociales 2019.

https://iabspain.es/estudio/estudio-anual-de-redes-sociales-2019

Kasitoko (2017). “¿Cómo diferenciar influencers, micro influencers y celebrities?”. Kasitoko.com.

https://kasitoko.com/como-diferenciar-influencers-micro-influencers-y-celebrities

Launchmetrics (2017). Informe sobre el estatus del marketing de influencers 2017.

https://www.launchmetrics.com/es/recursos/whitepapers/informe-marketing-influencers-2017

Launchmetrics (2018). Estatus del marketing de influencers en la moda, el lujo y la cosmética 2018.

https://www.launchmetrics.com/es/recursos/whitepapers/informe-marketing-de-influencers-2018

Launchmetrics (2019). The state of influencer marketing 2019. A special focus on fashion, luxury \& cosmetics. https://www.launchmetrics.com/landing/influencer-marketing-report-2019

Marwick, Alice E.; Boyd, Danah (2010). “I tweet honestly, I tweet passionately: Twitter users, context collapse, and the imagined audience". New media \& society, v. 13, n. 1, pp. 114-133.

http://www.tiara.org/wp-content/uploads/2018/05/Marwick_boyd_TweetHonestly.pdf

Nielsen (2015). Informe. Así confiamos en la publicidad.

https://bit.ly/2PxcUF3

Ochoa, Carlos (2015). "Muestreo no probabilístico: muestreo por bola de nieve”. Netquest, 30 junio.

https://bit.ly/2LGuC9K

Orihuela, José-Luis (2015). "Los nuevos paradigmas de la comunicación”. Chasqui. Revista latinoamericana de comunicación, n. 77, pp. 10-13.

https://doi.org/10.16921/chasqui.v0i77.1416 
Pedroni, Marco (2015). "'Stumbling on the heels of my blog': Career, forms of capital, and strategies in the (sub) field of fashion blogging". Fashion theory, v. 19, n. 2, pp. 179-199.

https://doi.org/10.2752/175174115X14168357992355

Pedroni, Marco (2016). "Meso-celebrities, fashion and the media: How digital influencers struggle for visibility". Film, fashion \& consumption, v. 5, n. 1, pp. 103-121.

https://doi.org/10.1386/ffc.5.1.103_1

Pedroni, Marco; Sádaba, Teresa; SanMiguel, Patricia (2017). "Is the golden era of fashion blogs over? An analysis of the Italian and Spanish fields of fashion blogging". In: Mora, Emmanuela; Pedroni, Marco (eds.). Fashion tales. Feeding the imaginary. Bern: Peter Lang, pp. 105-124. ISBN: 9783034327879

Pérez-Curiel, Concha; Clavijo-Ferreira, Lucía (2017). “Comunicación y social media en las empresas de moda”. Prisma social, n. 18, pp. 226-258.

https://revistaprismasocial.es/article/view/1440

Pérez-Curiel, Concha; Luque-Ortiz, Sergio (2017). "El marketing de influencia en moda. Estudio del nuevo modelo de consumo en Instagram de los millennials universitarios". AdComunica, n. 15, pp. 255-281.

https://doi.org/10.6035/2174-0992.2018.15.13

Pérez-Curiel, Concha; Sanz-Marcos, Paloma (2019). “Estrategia de marca, influencers y nuevos públicos en la comunicación de moda y lujo. Tendencia Gucci en Instagram". Prisma social, n. 24.

https://revistaprismasocial.es/article/view/2826

Posner, Harriet (2016). Marketing de moda. Barcelona: Gustavo Gili. ISBN: 9788425223761

Rocamora, Agnes; Bartlett, Djurdja (2009). "Fashion blogs: New spaces in fashion discourse”. Sociétés, v. 104, n. 2, pp. 105-114.

Ruiz-Molina, Encarna (2013). "Los blogs de moda en España: de la espontaneidad del usuario a la profesión de blogger". RUTA: Revista universitària de treballs acadèmics, n. 5, pp. 1-25.

https://www.raco.cat/index.php/Ruta/article/view/275935

SanMiguel, Patricia; Sádaba, Teresa (2019). "Consumo de información de moda entre los millennials". Prisma social, n. 24, pp. 186-206.

https://revistaprismasocial.es/article/view/2840

Socialbakers (2019). Mast-know influencer marketing trends.

https://www.socialbakers.com/blog/must-know-influencer-trends-for-2019-the-complete-report

Social Publi (2018). I Estudio de anunciantes con influencers.

http://recursos.anuncios.com/files/1028/24.pdf

Van-Dijck, José (2013). The culture of connectivity: A critical history of social media. Oxford: Oxford University Press. ISBN: 9780199970780

Wolfson, Courtney (2017). "Macro vs. micro influencers". Revolution digital, 30 January.

https://www.revolutiondigital.com/article/macro-vs-micro-influencers

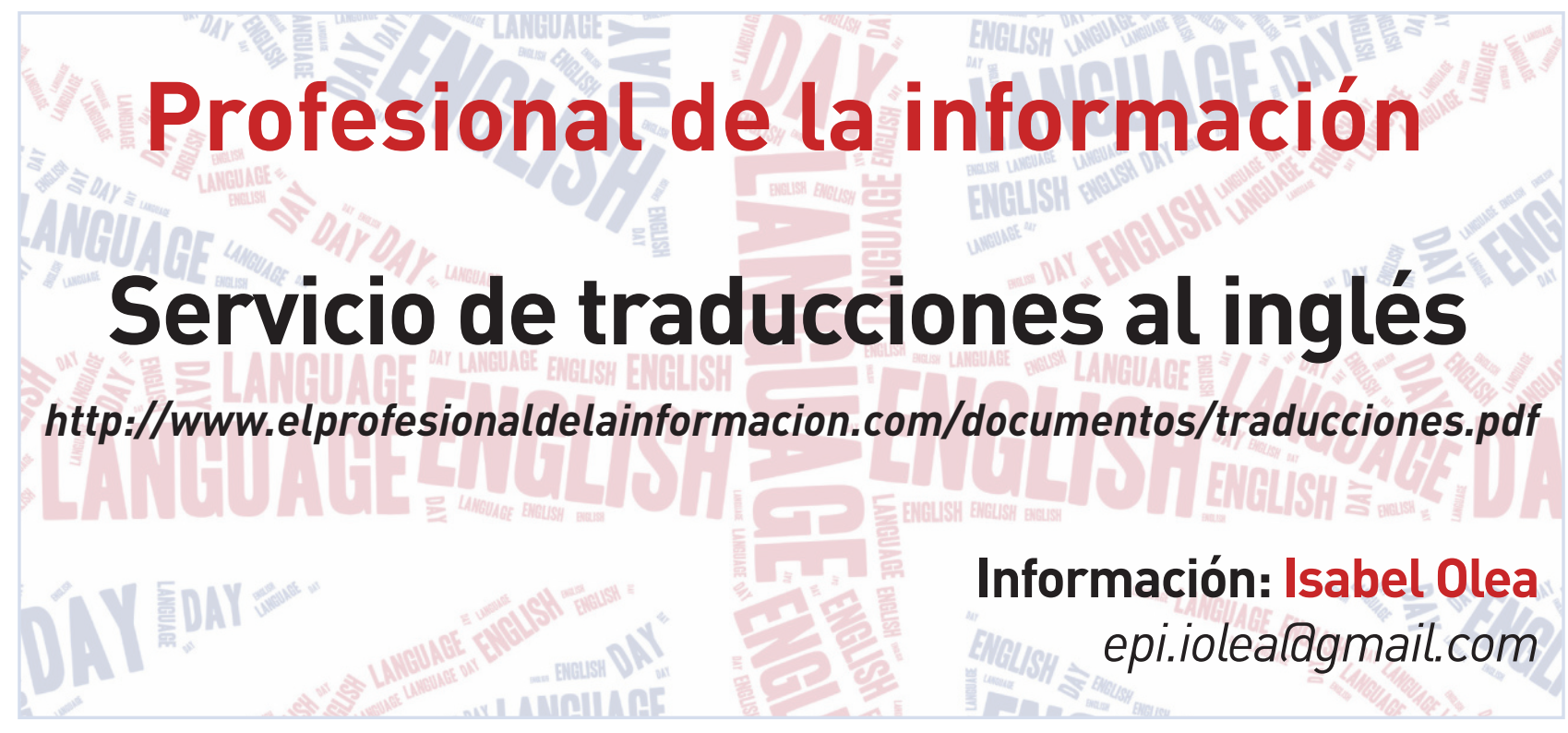

